\title{
Gentle Handling Attenuates Innate Defensive Responses to Visual Threats
}

\author{
Xuemei Liu' ${ }^{1,2 \dagger}$, Chen Chen ${ }^{1 \dagger}$, Yuanming Liu' ${ }^{1 \dagger}$, Zhijie Wang ${ }^{1}$, Kang Huang ${ }^{1}$, Feng Wang ${ }^{1 *}$ \\ and Liping Wang ${ }^{1 *}$
}

\begin{abstract}
1 Shenzhen Key Lab of Neuropsychiatric Modulation and Collaborative Innovation Center for Brain Science, CAS Center for Excellence in Brain Science and Intelligence Technology, The Brain Cognition and Brain Disease Institute, Shenzhen Institutes of Advanced Technology, Chinese Academy of Sciences, Shenzhen, China, ${ }^{2}$ University of Chinese Academy of Sciences, Beijing, China
\end{abstract}

\section{OPEN ACCESS}

Edited by:

Gérard Coureaud,

INSERM U1028 Centre de Recherche en Neurosciences de Lyon, France

Reviewed by:

Jeffrey B. Rosen,

University of Delaware, United States

Rafael S. Maior,

Universidade de Brasilia, Brazi

*Correspondence:

Feng Wang

feng.wang@siat.ac.cn

Liping Wang

Ip.wang@siat.ac.cn

tThese authors have contributed equally to this work

Received: 27 June 2018 Accepted: 24 September 2018

Published: 18 October 2018

Citation:

Liu X, Chen C, Liu Y, Wang Z Huang K, Wang F and Wang L (2018)

Gentle Handling Attenuates Innate Defensive Responses to Visual

Threats.

Front. Behav. Neurosci. 12:239. doi: 10.3389/fnbeh.2018.00239
Innate defensive responses to threats are essential for animal survival. The complexity and variability of innate defensive behaviors can be due to individual experiences, environmental factors, and internal states. However, it is not completely understood if the gentle handling involved in sensory processing affects innate defensive responses to visual threats. Here, we report attenuation of innate defensive responses after gentle handling accompanied by de-excitation of the intermediate layer (IL) and deep layer (DL) of the superior colliculus (SC) but not of the superficial layer (SL). Our theoretical analysis of the c-Fos network revealed an increased correlation in module 1, which maybe generally functionally associated with fear emotional, a decreased correlation in module 2, which maybe generally functionally associated with sensory processing. The IL of the SC appeared to have the highest correlation with the two modules. We verified the dynamic activities of the IL of SC in response to overhead looming stimulus using fiber photometry. Retrograde labeling of 18 regions of interest (ROIs) showed that the IL received significant inputs from the cortical areas, thalamus, hypothalamus, and brainstem. These data suggest the sensory processing involved in the modulatory roles of the SC in innate fear processing.

Keywords: gentle handling, innate fear, sensory input, superior colliculus, overhead looming stimulus

\section{INTRODUCTION}

Innate defensive responses to threats are essential for animal survival (LeDoux, 2012; Anderson and Adolphs, 2014; Janak and Tye, 2015; Tovote et al., 2015). Although defensive behaviors are stereotyped, their complexity and variability can be due to individual experiences (Morgan, 1896), environmental factors, and internal states (LeDoux, 2012; Anderson and Adolphs, 2014; Anderson, 2016). However, whether sensory input regulate the innate fear behavioral output is not well defined.

Overhead looming stimuli that mimic aerial predators have been shown to trigger stereotyped defensive responses across species (Yilmaz and Meister, 2013; Muijres et al., 2014; Temizer et al., 2015). Recently, looming-evoked defensive behavior and its underlying neural circuitry via the superior colliculus (SC) have received increasing attention (Yilmaz and Meister, 2013; Shang et al., 2015; Wei et al., 2015; De Franceschi et al., 2016; Huang et al., 2017; Vale et al., 2017; Evans et al., 2018; Li et al., 2018; Salay et al., 2018). SC is a laminated retinal 
recipient structure, of which its superficial layer (SL) receives direct retina input and primarily responds to visual stimuli. The intermediate layer (IL) and deep layer (DL) of the SC contain neurons that respond to multimodal (somatosensory, auditory, visual) sensory inputs (Wurtz and Goldberg, 1971; Stein, 1984).

Laboratory housing, husbandry, and handling methods have been shown to affect the internal state of the animals (Grandin, 1997; Poole, 1997; Deacon, 2006; Hurst and West, 2010; Clarkson et al., 2018). Unpleasant somatosensations such as tail handling and rough handling reduce the response to reward and increase stress levels (Grandin, 1997; Clarkson et al., 2018). Conversely, a gentle touch plays an essential role in the pleasant somatosensory processing conserved across species (Lumpkin et al., 2010; Abraira and Ginty, 2013). In mammals, a gentle touch is the expression of preference between mates, parenting, and affiliation (Barnett, 2005; Ebisch et al., 2016). It has also been demonstrated that massage has positive effects on health in human, grooming reduces stress levels in primates, and tactile stimulation alleviates the effects of stress in fish (Field et al., 2005; Soares et al., 2011). Our previous study demonstrated that stress accelerates the defensive responses to looming in mice (Li et al., 2018). However, it remains unknown if the repeat pleasant somatosensation of gentle handling affects innate defensive behaviors to visual threats.

Here, we found that gentle handling attenuated innate defensive responses to overhead looming. In addition, the IL and DL (but not the SL) of the SC had significantly reduced activities in response to overhead looming after repeated gentle handling. Functional analysis of the c-Fos network revealed that the highest correlation was between the IL of the SC in two modules among the 16 ROIs. Moreover, we verified the dynamic activities of the IL of SC to overhead looming stimulus using fiber photometry. Retrograde labeling of 18 ROIs showed that the IL had significant inputs from the cortical areas, thalamus, hypothalamus, and brainstem, suggesting the potential modulatory roles of the SC in innate fear and sensory processing.

\section{MATERIALS AND METHODS}

\section{Animals}

All husbandry and experimental procedures in this study were approved by the Animal Care and Use Committee at the Shenzhen Institute of Advanced Technology, Chinese Academy of Sciences (Shenzhen, China). Male (6 weeks of age) C57BL/6 mice obtained from Beijing Vital River Laboratory Animal Technology Co., Ltd. (Beijing, China) were kept in a quiet room $\left(22-25^{\circ} \mathrm{C}\right)$ with a $12 \mathrm{~h}$ light-dark cycle, and were given food and water ad libitum.

\section{Viral Injection}

Mice were anesthetized with intraperitoneal (i.p.) injection of pentobarbital sodium $(0.5 \% \mathrm{w} / \mathrm{v}, 80 \mathrm{mg} / \mathrm{kg})$ and placed in a stereotaxic frame (RWD Life Science, Shenzhen, China). Microinjection needles were inserted unilaterally directly above the IL of the SC, using the following coordinates from Bregma: $\mathrm{AP},-3.85 \mathrm{~mm}, \mathrm{ML}, \pm 0.8 \mathrm{~mm}$, and $\mathrm{DV},-1.85 \mathrm{~mm}$. The cholera toxin subunit B (CTB) Alexa Fluor 594 Conjugate and adenoassociated virus (AAV) expressing EGFP driven by a neuron calcium/calmodulin-dependent kinase II (CaMKII) promoter (AAV9-CaMKII $\alpha$-GCaMP6-EGFP; BrainVTA Co., Ltd., China) were injected using a $10 \mu \mathrm{L}$ microsyringe with a 33 gauge metal needle (Hamilton; Sigma, United States) connected to the UltraMicroPump 3 microsyringe pump injector (World Precision Instruments, United States) and its controller (Micro4; World Precision Instruments) at a rate of $100 \mathrm{~nL} / \mathrm{min}$. After injection, the needle was left in place for an additional $10 \mathrm{~min}$ to allow diffusion of the virus particles away from the injection site, and then it was slowly withdrawn.

\section{Histology, Immunohistochemistry, and Microscopy}

To quantify the expression of c-Fos positive neurons in the whole brain induced by overhead looming stimuli after consecutive 7 days of gentle handling or no-handled, the two group of mice were sacrificed $1.5 \mathrm{~h}$ after presentation of overhead looming stimuli on the 8 th day. Mice were perfused by overdosing with chloral hydrate (10\% w/v, $300 \mathrm{mg} / \mathrm{kg}$, i.p.) and transcranially perfused with cold $1 \mathrm{M}$ PBS followed by ice-cold $4 \%$ paraformaldehyde (PFA; Sigma) in $1 \mathrm{M}$ PBS. Brains were removed and submerged in $4 \% \mathrm{PFA}$ at $4^{\circ} \mathrm{C}$ overnight to postfix, and then transferred to $30 \%$ sucrose to equilibrate. The coronal brains sections $(40 \mu \mathrm{m})$ were obtained with a cryostat microtome (CM1950; Leica, Germany). Freely floating sections were washed with PBS, and blocked for $1 \mathrm{~h}$ at room temperature in blocking solution containing $0.3 \%$ Triton X-100 and 10\% normal goat serum (NGS). Then the sections were incubated overnight with rabbit monoclonal anti-c-Fos (1:300, \#2250; Cell Signaling Technology, United States) diluted in PBS with 3\% NGS and $0.1 \%$ TritonX-100. The sections were incubated for $1 \mathrm{~h}$ at room temperature with Alexa Fluor 488 goat anti-rabbit secondary antibody (1:200; Jackson Laboratory, United States). Finally, the sections were mounted, coverslipped with DAPI (1:50,000, \#62248; Thermo Fisher Scientific, United States), and photographed using the Olympus VS120 virtual microscopy slide scanning system (Olympus; Japan). The images were acquired using identical gain and offset settings, and analyzed with ImageJ, Image Pro Plus, and Adobe Photoshop software. ROIs were traced with reference to the "The mouse brain in stereotaxic coordinates, George Paxinos and Keith B. J. Franklin," and c-Fos immunoreactivity was quantified using Image Pro Plus that was checked by comparing to manual counts by a trained doubleblind observer.

\section{Behavior Assay Gentle Handling}

After obtained from the laboratory animal center, the mice have 1 week to habituation to the new animal facility and cage homes. Gentle handling was performed in the arena.

Briefly, mice were placed individually on a piece of medical absorbent cotton for $3 \mathrm{~min}$ handling once per day for 7 consecutive days. Handling sessions were performed by gently and thoroughly touching the mouse body through the cotton. 
New cages with the previous bedding were used to house the mice that had been handled. Handling sessions were performed in the same room by the same experimenter. The mice in the nonhandled group received the same approach without the handling treatment, including habituation to the looming box on the 7 th day and receive three times upper visual looming stimuli on the 8 th day.

\section{Looming Stimulation Test}

The behavioral arena was a $40 \mathrm{~cm} \times 40 \mathrm{~cm} \times 30 \mathrm{~cm}$ closed plexiglass box with a shelter nest in the corner and an LCD monitor on the ceiling to present the looming stimulus (LS). The LS was a black disk that expanded from 2 to 20 degrees in diameter; it was presented 15 times in $5.5 \mathrm{~s}$. The stimulation was manually triggered by the experimenter when the mice were in the farthest area away from the shelter. The handling group of the mice have experienced the recurrent 7 days gentle handling. On the 7th day, the mice in gentle handled group and nonhandled group have $10 \mathrm{~min}$ to habituation to the looming box. Then on the 8 th test day, after 3 min pre-test session, all the mice have subjected three times of the upper visual black looming stimulation. And the duration between each of the stimulation is no less than $3 \mathrm{~min}$.

\section{Fiber Photometry}

A fiber photometry system (ThinkerTech, Nanjing) was used to record the calcium signals of $\mathrm{CaMKII} \alpha$-positive neurons in the IL of the SC in response to upper visual field looming stimulation. Two to 3 weeks following AAV9-CaMKII $\alpha$-GCaMP6-EGFP injection into the IL, an optical fiber (220 $\mu \mathrm{m}$ O.D., $0.37 \mathrm{NA}$; Newdoon, China) was placed above the IL of the SC. One week after the surgery, mice took $10 \mathrm{~min}$ to habituation to looming box the day before the test day. In the test day, mice received three times upper visual field black looming stimulations and three times upper visual field white looming stimulations. The upper visual field white looming here is used as a control which does not evoke the flight behavior.

To record the fluorescence signals, a $480 \mathrm{~nm}$ excitation light from a LEDs (CREE XPE), reflected of a dichroic mirror with a 435-488 nm reflection band and a 502-730 $\mathrm{nm}$ transmission band (Edmunds Inc.) and coupled to a long optical fiber $(220 \mu \mathrm{m}$ O.D., 0.37 NA, $2 \mathrm{~m}$ long, Thorlabs, Inc.). The laser intensity at the fiber tip was $20-30 \mu \mathrm{W}$ to minimize GCaMP bleaching. GCaMP fluorescence was filtered with a GFP bandpass (Filter 525/39; Thorlabs, Inc., United States), detected by the sensor of a CMOS camera (Thorlabs, Inc. DCC3240M). A Lab view program is developed to control the CMOS camera and acquire calcium signal in about $50 \mathrm{~Hz}$. The behavior event signal is recorded by a DAQ card (NI, usb-6001) in $1000 \mathrm{~Hz}$ using the same program.

\section{Statistical Analysis}

All data values are presented as the mean \pm SEM. MannWhitney $U$ test was used to analyze statistical differences using GraphPad Prism 7 software. Statistical significance was set at ${ }^{*} p<0.05,{ }^{* *} p<0.01,{ }^{* * *} p<0.001,{ }^{* * * *} p<0.0001$. Functional network analyses of the c-Fos dataset were computed with the Rubinov and Sporns (2010) Brain Connectivity Toolbox ${ }^{1}$ in MATLAB R2016 (The Mathworks Inc.) Graph theoretical analysis of c-Fos data between gentle handling and non-handling conditions was used to calculate the relationship among the 16 ROIs. Correlations among the 16 ROIs arranged by rankorder were conducted using Kendall's Tau correlation coefficient. Community correlation network and participation coefficients were analyzed according to previous studies, and a threshold value of 0.35 was set (Newman, 2006; Power et al., 2013; Vetere et al., 2017; Rogers-Carter et al., 2018).

\section{RESULTS}

\section{Gentle Handling Attenuates Defensive Responses to Overhead LS}

After 1 week habituation in the laboratory, mice underwent 7 consecutive days of gentle handling. To determine whether gently handling affects the innate defensive responses of mice, we used a behavioral assay with a rapidly expanding dark disk stimulus that mimicks an approaching predator (Yilmaz and Meister, 2013; Shang et al., 2015; Wei et al., 2015; De Franceschi et al., 2016; Huang et al., 2017; Vale et al., 2017; Salay et al., 2018). In response to repeated LS (three trials, intervals longer than $3 \mathrm{~min}$ ), gently handled mice had an increased latency to onset flight behavior (Figure 1B), increased latency of flight to nest (Figure 1C), decreased duration hiding in nest (Figure 1D), and decreased locomotion speed (Figure 1E) compared with the non-handled group $\left(n=12\right.$ in each group, Mann-Whitney $U$ test, ${ }^{*} p<0.05$, $\left.{ }^{* *} p<0.01,{ }^{* * *} p<0.001,{ }^{* * * *} p<0.0001\right)$. Taken together, these results demonstrate that long-term repeated handling attenuates innate defensive responses to visual threat stimuli. These data suggest that instinctive behavioral output is sensitive to tactile stimulation.

\section{Gentle Handling Reduces the Activities of SC Neurons in Response to Overhead Looming Stimulus}

To test whether gentle handling affects SC responses to LS, we performed c-Fos labeling in the gently handled and non-handled groups after the behavioral test. The gently handled group had attenuated innate defensive behavior (Figure 1) accompanied by a decrease of c-Fos expression in the IL and DL of the SC, but not in the SL of the SC (Figure 2; $n=6$ mice in each group, MannWhitney $U$ test, $U=8, p=0.132, U=2,{ }^{* *} p=0.0087, U=4$, $* p=0.0260)$. These data indicate that gentle handling reduced the activities of the IL and DL of the SC in response to the black looming in the upper visual field.

\section{Analyses of the c-Fos Functional Network in Response to Overhead Looming Stimulus After Gentle Handling}

To determine the functional network connectivity, we performed c-Fos mapping in 16 ROIs in response to overhead LS between

\footnotetext{
${ }^{1}$ https://sites.google.com/site/bctnet/
} 
A
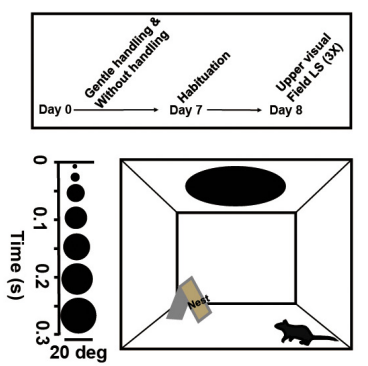

C

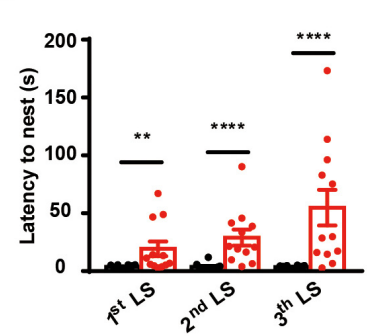

B

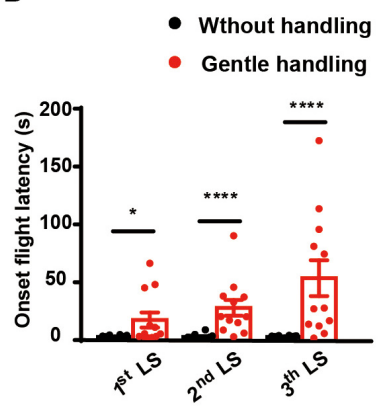

D
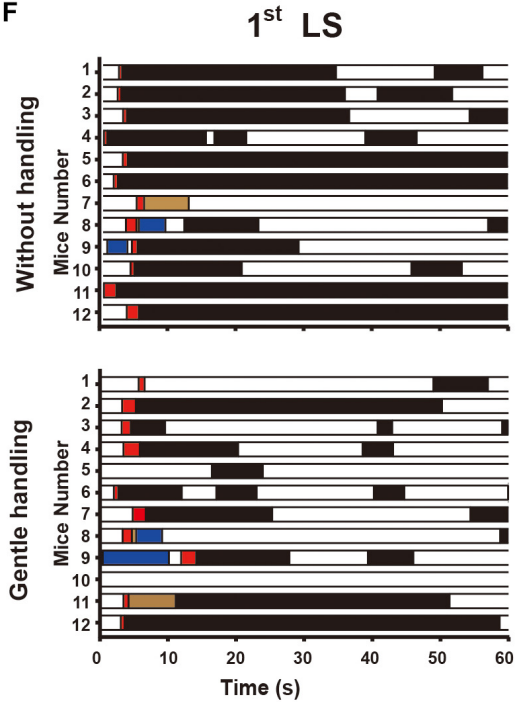

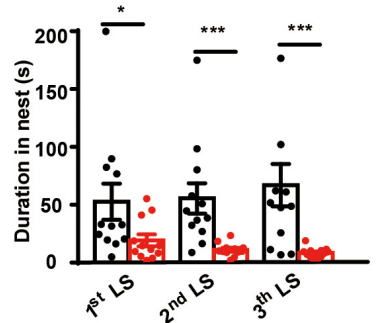

E

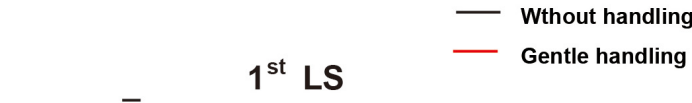

Whand

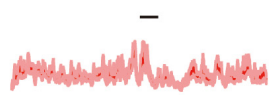

$2^{\text {nd }}$ LS

$1^{\text {st }}$ LS

Wthout handling

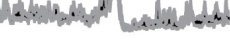
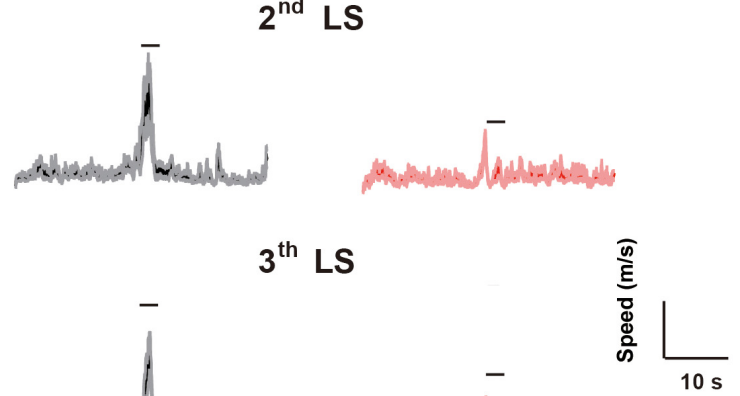

$0 \mathrm{~s}$

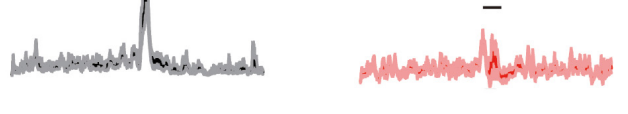

- Nest $\odot$ Flight $\odot$ Freezing $\triangle$ Corner $\bigcirc$ Out

LS
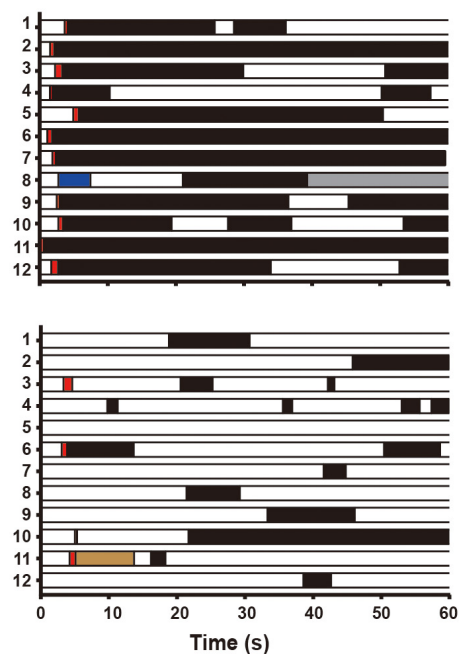

$3^{\text {th }}$ LS
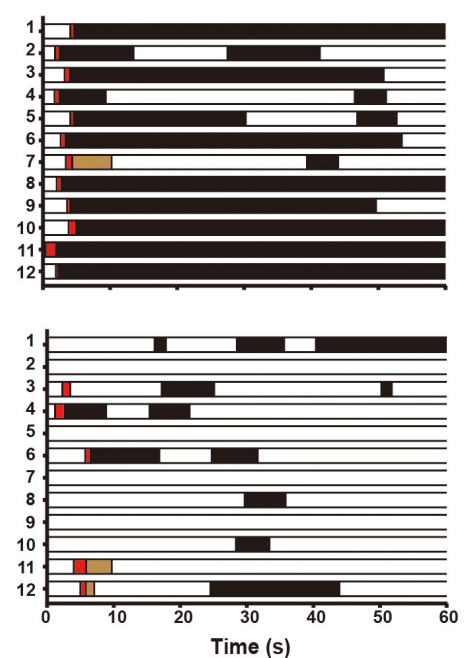

FIGURE 1 | (A) Schematic diagram showing the behavioral arena and dark disk expansion from 2-20 degrees for 15 repeated cycles in 5.5 s. Timeline showing the design of the gentle handling method (days 1-7, $3 \mathrm{~min} /$ day). On the test day (day 8), LS was presented for 15 repeated cycles in $5.5 \mathrm{~s}$ with intervals of at least 3 min. After three trials with overhead LS, Mann-Whitney $U$ tests showed that the gently handled group had longer latency to (B) onset flight behavior $(U=31, p=0.0165$; $U=5, p<0.0001 ; U=9, p<0.0001$ after 1st, 2nd, and 3rd LS, respectively) (C) latency of flight to nest $(U=21, p=0.0022 ; U=5, p<0.0001 ; U=6.5$, $p<0.0001$ after 1st, 2 nd, and 3rd LS, respectively), and (D) decreased duration hiding in the nest $(U=32, p=0.0205 ; U=12, p=0.0002 ; U=17, p=0.0008$ after 1st, 2nd, and 3rd LS, respectively) compared with the non-handled group ( $n=12$ in each group; $\left.{ }^{*} p<0.05,{ }^{* *} p<0.01,{ }^{* * *} p<0.001,{ }^{* * * *} p<0.0001\right)$. Data are presented as the mean \pm SEM. (E) The average locomotion speed of mice with gentle handling in the three trials of LS (horizontal black bar indicates duration of looming, $n=12$ mice in each group, shaded area indicates SEM of the averaged data before and after $60 \mathrm{~s})$. (F) Occurrence of flight, freezing, hiding in nest, and out of nest behaviors 1 min after 1st, 2nd, and 3rd LS presentation.

gently handled and non-handled mice. The 16 ROIs are selected by the expression of c-Fos which is comparatively higher than some other brain regions, also the brain regions what we choose, is most like generally functionally associated with the "fear" and "sensory" processing. Graph theoretical analysis was used to divide the 16 ROIs into two modules, in which the functional correlation of ROIs in each module was higher than that between modules (Figure 3A).

Our paradigm used in the current study is overhead lifethreatening looming stimulation which mimic the predatory in the upper visual field. And most ROIs in module 1 (Figure 3D, purple dots) maybe generally functionally associated 
A

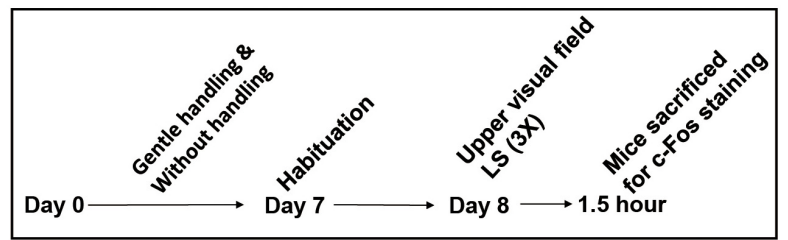

- Without handling

- Gentle handling

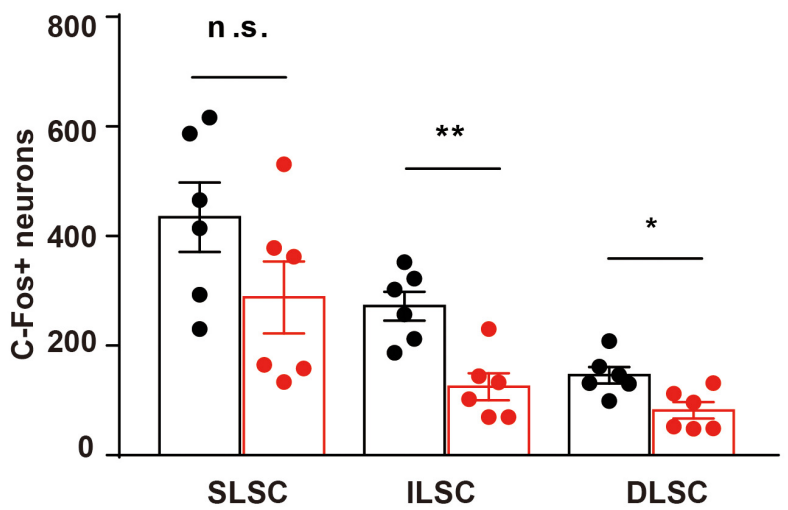

B

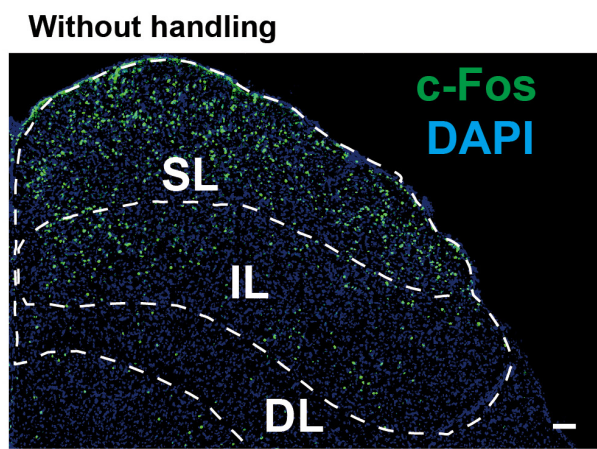

Gentle handling

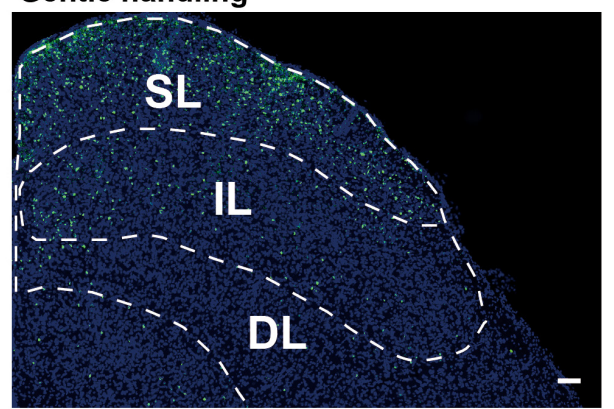

FIGURE 2 | (A) The timeline and bar graph showing reduced c-Fos expression in the gently handled group compared with the non-handled group in response to LS. (B) Representative images showing C-Fos expression in the SC of gently handled or non-handled groups in response to LS (SL, superficial layer of SC; IL, intermediate layer of SC; DL, deep layer of SC); Scale bars = $100 \mu \mathrm{m}$. Mann-Whitney $U$ test, ${ }^{* *} P<0.01, U=2 ; * P<0.05, U=4$.

with "fear" related, such as different layer of superior colliculus, ventral tegmental area, basolateral amygdala, anterior cingulate cortex, pontine gray, piriform cortex. And the recurrent longterm (7 days continuously stimulation) gentle handling should transmit sensory input signal. And most ROIs in module 2 (Figure 3D, green dots) maybe generally maybe generally functionally associated with "sensory" related, such as primary somatosensory cortex, barrel field; primary somatosensory cortex, trunk region; primary visual cortex. After gentle handling, the correlation was decreased in module 1 and increased in module 2, and the correlation across modules 1 and 2 was increased (Figure 3B). The participation coefficients were calculated to determine the hubs in the functional correlationbased networks (Power et al., 2013; Vetere et al., 2017; RogersCarter et al., 2018). The nodes with the highest participation coefficients across two modules included brain areas such as the IL and DL of the SC, S1BF, V1, PVT, and PAG (Figure 3C). Functional network analysis indicated that compared to the SL and DL, the IL of the SC was more densely connected with other nodes related to innate fear and sensory perception.

\section{Dynamic Activation of the IL of SC in Response to Looming Overhead Stimulus}

The SC, especially the SL of the SC, receives direct retinal input and mediates visual threat processing (Shang et al.,
2015, 2018). To investigate whether the IL of the SC receiving somatosensory input reflects visual threat signals, we injected AAV9-CaMKII $\alpha$-GCaMP6s into the SC and optical fibers were implanted into the IL. Fiber photometry was used to record calcium transients in CaMKII $\alpha$-positive neurons in the IL in response to a black looming in the upper visual field (Figure 4A). A white looming in the upper visual field was used as a control, as it does not trigger flight-to-nest behavior (Figures 4B,C). The IL of SC CaMKII $\alpha$-positive neurons exhibited a significant increase in the activity after presentation of a black LS in the upper visual field $(9.52 \pm 2.13 \%, \Delta \mathrm{F} / \mathrm{F}$ mean) compared with the white LS in the upper visual field $(0.98 \pm 0.74 \%, \Delta \mathrm{F} / \mathrm{F}$ mean $)$. Calcium signal onset to looming overhead stimulus rapidly increased $(15 \%$ of peak signal as the onset time point) with a latency of $0.71 \pm 0.18 \mathrm{~s}$ and peak signal of $19.08 \pm 3 \%, \Delta \mathrm{F} / \mathrm{F}$ (Figures 4D,E). Taken together, these data demonstrate the IL of SC CaMKII $\alpha$-positive neurons encode visual threat processing.

\section{Retrograde Labeling of Input to the IL of the SC}

To visualize inputs to the IL, the CTB Alexa Fluor 594 Conjugate CTB was used for tracing. Two weeks after microinjection of CTB into the IL, the brain was sectioned to $40 \mu \mathrm{m}$, and every third section was processed for subsequent analysis. Across the entire brain, the most abundant labeling was found in the cortex (Aux, V1, S1, ACC, TeA, EcT, PRh, EnT), thalamus (LD, LP, AV), 
A

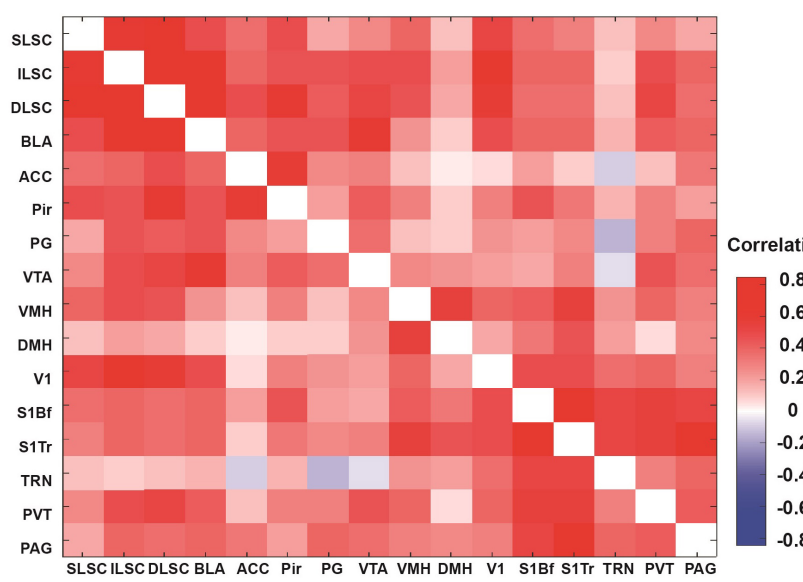

C

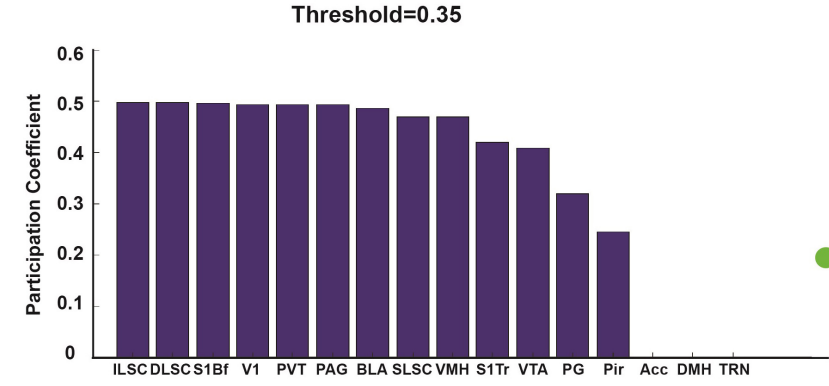

政
B
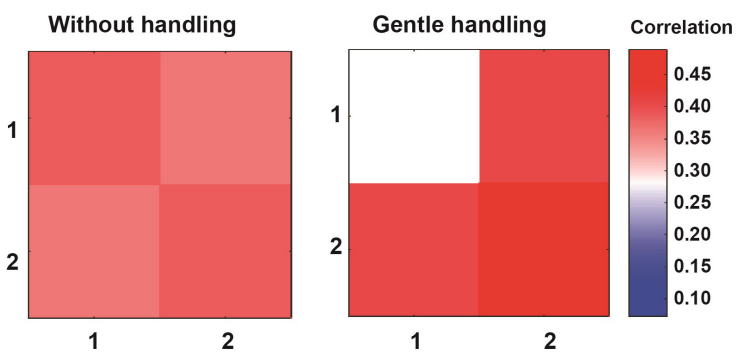

Module 1

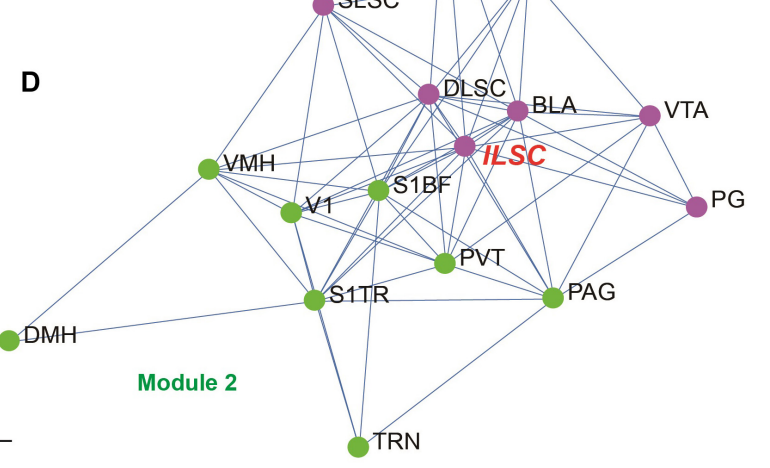

FIGURE 3 | (A) Correlation matrix showing the functional correlation among ROls, SLSC (superficial layer of superior colliculus), ILSC (intermediate layer of superior colliculus ), DLSC (deep layer of superior colliculus), BLA (basolateral amygdala), ACC (anterior cingulate cortex), Pir (piriform cortex), PG (pontine gray), VTA (ventral tegmental area), VMH (ventromedial hypothalamic nucleus), DMH (dorsal hypothalamic area), V1 (primary visual cortex), S1BF (primary somatosensory cortex, barrel field), S1TR (primary somatosensory cortex, trunk region), TRN (tegmental reticular nucleus), PVT (paraventricular thalamic nucleus), PAG (periaqueductal gray matter). (B) Two modules were classified by graph theoretical analysis. (C) Participation coefficients were calculated to determine the degree of each node connected to multiple networks. (D) Functional network nodes were exhibited by network visualization (module 1 is in purple, module 2 is in green).

and hypothalamus (LH, DMH, VMH, ZI), brainstem (LC, DRN) (Figure 5). These data suggest that the IL of the SC receives broad cortical inputs from visual cortex, auditory cortex, and somatosensory cortex, as well as autonomic inputs from LC, LH, and thalamic nuclei (Saper, 2002).

\section{DISCUSSION}

The innate defensive reactions to threats are vital for survival. Although innate fear is instinctive and unconditional, the expression of innate fear can be affected by the circuits of motivation, arousal, emotional state, and reinforcement (LeDoux, 2012; Anderson and Adolphs, 2014). Tactile stimuli is a fundamental form of sensory perception conserved across species (Ratcliffe, 2012). Gentle handling has been shown to reduce stress, modify cognitive behavior, and improve the relationship between handlers and animals (Harlow and Suomi, 1970; Antoniazzi et al., 2017). Furthermore, gentle handling has been proven to decrease depression and reduce anxietylike behavior in laboratory mice (Grandin, 1997; Madruga et al., 2006). Visually evoked defensive responses to overhead looming stimulus are initiated from the SC and lay downstream of the SC
(Wei et al., 2015; Shang et al., 2018), but whether gentle handling affects innate visual threat processing is largely unknown.

In the current study, we demonstrated that gentle handling attenuates innate defensive reactions to visual threat stimuli, consistent with findings from previous studies (Grandin, 1997; Madruga et al., 2006). These studies provide a possibility that gentle handling might change the social interaction with experimenter and animal to improve the animal's performance. Moreover, it's possible that gentle handle induced the neurotransmitters such as oxytocin and dopamine release or lower corticotropin releasing hormone $(\mathrm{CRH})$ to reduce emotionality, promote calm, and increase the ability to address visual threat situation. (Harlow and Suomi, 1970; Fenoglio, 2006; Ellingsen et al., 2014; Scheele et al., 2014; Peled-Avron et al., 2016).

Our c-Fos network data analysis revealed that after gentle handling, the correlation became weaker in module 1 , but was stronger in module 2, and the functional connection across modules 1 and 2 became much stronger, as seen in Figure 4B. This suggests that gentle handling reduces activity in fear emotional-related regions but enhances the sensory connection response to visual threat. Functional network analysis demonstrated the IL and DL of the SC, S1BF, V1, PVT, and 


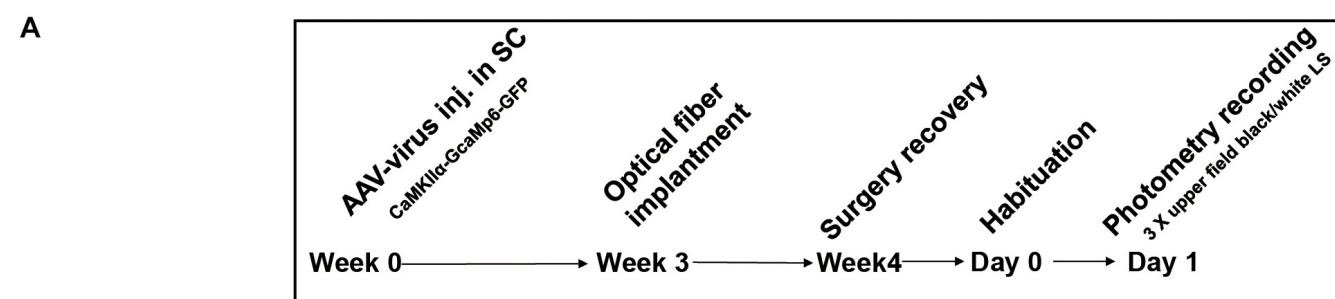

B

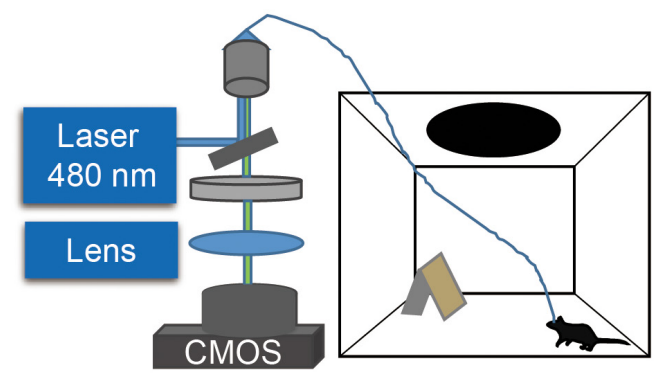

D

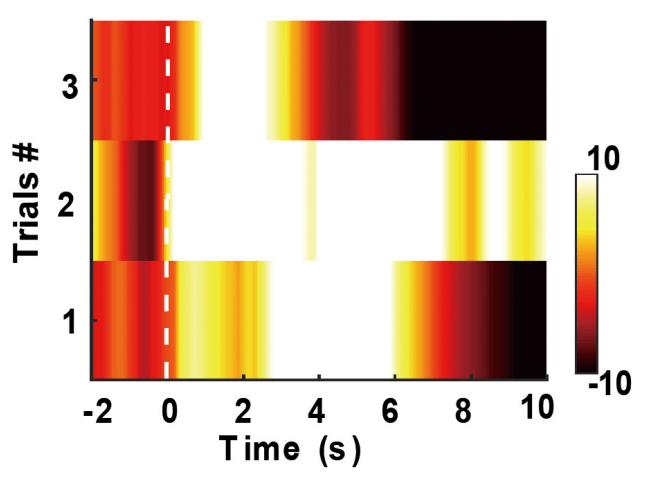

C

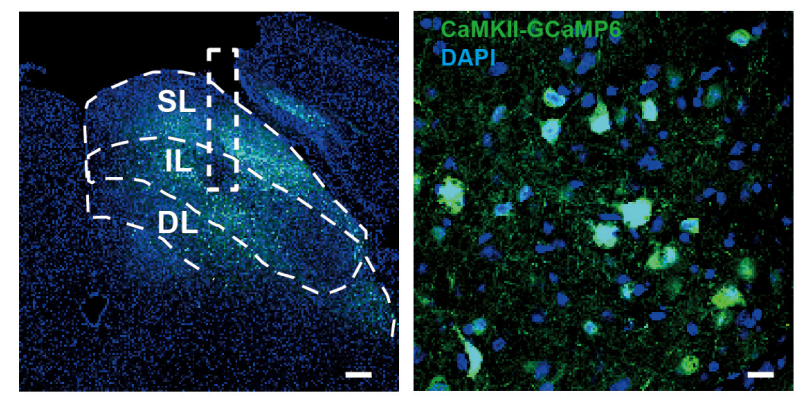

- Upper field black LS

- Upper field white LS
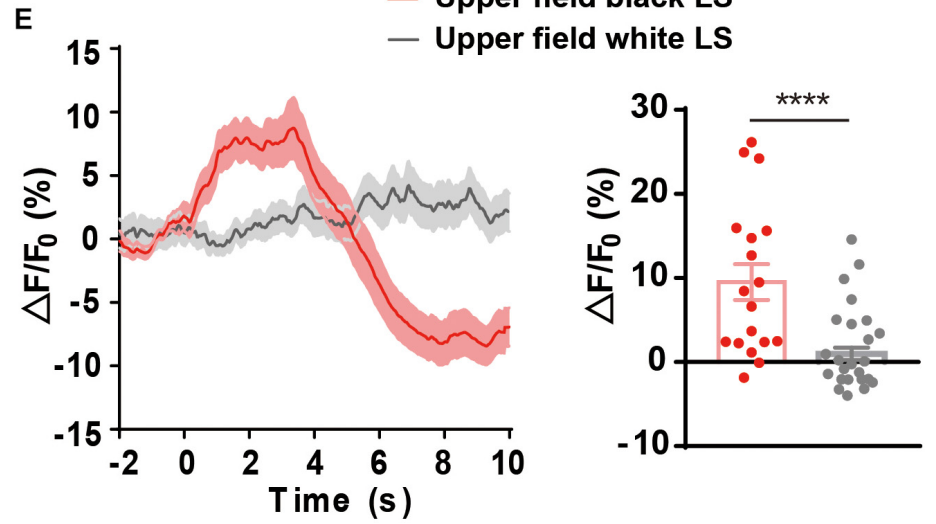

FIGURE 4 | (A,B) The timeline and schematic of fiber photometry recording of LS-induced Ca ${ }^{2+}$ transient. (C) AAV9-CaMKIl $\alpha$-GCaMP6-GFP expression in the IL of the SC; Scale bars, left $=100 \mu \mathrm{m}$; right $=10 \mu \mathrm{m}$. (D) Representative heatmap exhibits LS-induced Ca ${ }^{2+}$ transient across three trials. (E) Mean GCaMP signal response to black looming in the upper visual field and white looming $\left(n=6\right.$ mice in black looming, $n=5$ mice in white looming, ${ }^{* * * *} p<0.0001$, Mann-Whitney $U$ test, $U=117$ ).

PAG, these brain areas have the highest participation coefficients across two modules. IL and DL of SC are thought to be involving in defensive behavior and are also functionally as the multisensory integration center (Sahibzada et al., 1986; Mooney et al., 1992). And in our study, IL and DL of SC are vital hubs to process both the sensory and visual threatening signals. S1BF and V1 are a part of primary sensory cortex, previous studies have defined the S1BF encode the representation of a sensory stimulus shaped by associative fear learning and V1 inputs to SC increase the response magnitude to looming (Gdalyahu et al., 2012; Zhao et al., 2014). Our recurrent gentle handling supposed to enhance the cortical plasticity and change the connection between cortex and subcortex which could lead to a change of defensive responses respond to overhead looming. PVT is a part of thalamus, which is recognized as relay station to transfer the sensory information, has proved to contribute to threatening events and fear memory (Penzo et al., 2015). PAG has defined to orchestrates sensory and motor (Koutsikou et al., 2015) and initiation of escape to looming stimuli (Evans et al., 2018). Taken together, all these brain regions functionally as hubs in powerful way in sensory and fear related networks.

The SC is a laminate sensory-motor structure. The SL of SC is thought to process primarily visual signal, whereas the IL and DL of SC exert sensory responses and multimodal integration, contributing to saccadic eye movements and head movements (Wurtz and Goldberg, 1971; Stein, 1984). Our CTB retrograde tracing data indicated that the IL of the SC receives broad cortical and autonomic inputs. Another possible mechanism may be multimodal integration of the IL-SC, in which recurrent somatosensory stimuli enhance visual discrimination (Macaluso et al., 2000, 2002). Our c-Fos mapping data demonstrated that, gentle handling affects visual threat signals by preferentially influencing different layer of SC. That's c-Fos expression in gently handled mice was significantly higher in the IL and DL of the SC but not in the SL of the SC compared with the non-handled group respond to overhead looming stimuli. 

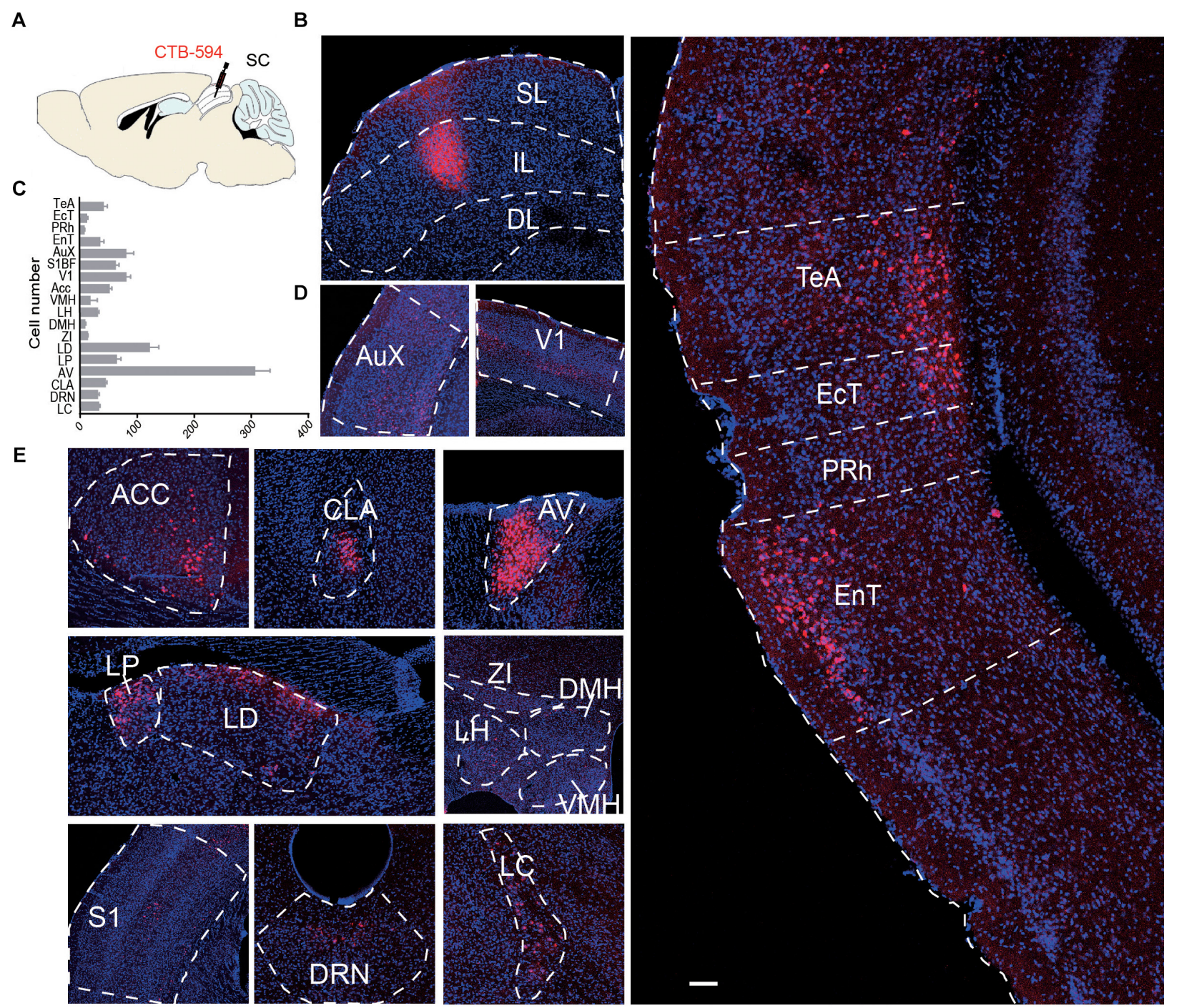

FIGURE 5 | (A) Schematic diagram and (B) representative image of CTB expression in the SL and IL of the SC. (C) Bar graph showing the number of input neurons in each area $(n=4)$; $(\mathbf{D}, \mathbf{E})$ Representative area of input neurons. AuX, auditory cortex; $\mathrm{V} 1$, primary visual cortex; ACC, anterior cingulate cortex; CLA, claustrum; AV, Anteroventral thalamic nucleus; LP, lateral posterior thalamic nucleus; LD, laterodorsal thalamic nucleus; ZI, zona incerta; VMH, ventromedial hypothalamic nucleus; LH, lateral hypothalamic area; DMH, dorsal hypothalamic area; S1, primary somatosensory cortex; DRN, dorsal raphe nucleus; LC, locus coeruleus; TeA, temporal association cortex; EcT, ectorhinal cortex; PRh, perirhinal cortex; EnT, entorhinal cortex. Scale bars = $250 \mu \mathrm{m}$.

In addition, our c-Fos functional network analysis confirmed that the IL has more intensive connections with the other regions than the DL and SL of the SC. However, the different functions of IL and DL have not been well defined. Thus, the neural mechanisms underlying interlaminar modulation of the SC needs further investigations (Mooney et al., 1992; Saito and Isa, 2005; Cohen and Castro-Alamancos, 2010; Ghitani et al., 2014).

The results of this study indicate that innate defensive responses are sensitive to external conditions, which are vital factors that influence animals behaviors by affecting the internal state of brain. Our data consistent with the previous studies suggest that the environmental condition (e.g., housing, husbandry, habituation, handling, transport) and standard of behavioral protocol are crucial for animal behavioral tests, especially the innate behavioral test (Grandin, 1997; Poole, 1997; Deacon, 2006; Baumans, 2009; Hurst and West, 2010; Clarkson et al., 2018).

Our current study revealed that gentle handling changes the activation of key brain regions that respond to danger signaling, indicating that these regions are vital for processing the interaction between sensory perception and fear emotion. The fact that recurrent sensory stimuli affect innate fear processing suggest that related neural circuits may be causally responsible for psychiatric diseases such as autism, of which the core characteristics are sensory and emotion deficits (Pernon et al., 2007; Cascio et al., 2012; Robertson and Baron-Cohen, 2017). 


\section{AUTHOR CONTRIBUTIONS}

XL, LW, and FW designed the experiments. CC performed the behavioral tests. YL conducted the fiber photometry recording. $\mathrm{CC}, \mathrm{YL}$, and $\mathrm{XL}$ performed the histological studies. XL, CC, YL, $\mathrm{ZW}$, and $\mathrm{KH}$ analyzed the data. XL, LW, and FW wrote the manuscript.

\section{FUNDING}

This work was supported by the National Natural Science Foundation of China (Grant Nos. NSFC 31630031, 81425010,

\section{REFERENCES}

Abraira, V. E., and Ginty, D. D. (2013). The sensory neurons of touch. Neuron 79, 618-639. doi: 10.1016/j.neuron.2013.07.051

Anderson, D. J. (2016). Circuit modules linking internal states and social behaviour in flies and mice. Nat. Rev. Neurosci. 17, 692-704. doi: 10.1038/nrn.2016.125

Anderson, D. J., and Adolphs, R. A. (2014). A framework for studying emotions across species. Cell 157, 187-200. doi: 10.1016/j.cell.2014.03.003

Antoniazzi, C. T. D., Metz, V. G., Roversi, K., Freitas, D. L., Vey, L. T., Dias, V. T., et al. (2017). Tactile stimulation during different developmental periods modifies hippocampal BDNF and GR, affecting memory and behavior in adult rats. Hippocampus 27, 210-220. doi: 10.1002/hipo.22686

Barnett, L. (2005). Keep in touch: the importance of touch in infant development. Infant Obs. 8, 115-123. doi: 10.1080/13698030500171530

Baumans, V. (2009). The impact of light, noise, cage cleaning and in-house. Lab. Anim. 43, 311-327. doi: 10.1258/la.2009.0080098

Cascio, C. J., Moana-Filho, E. J., Guest, S., Nebel, M. B., Weisner, J., Baranek, G. T., et al. (2012). Perceptual and neural responses to affective tactile texure stimulation in Adults with Autism Spectrum Disorders. Autism Res. 5, 231-244. doi: 10.1002/aur.1224

Clarkson, J. M., Dwyer, D. M., Flecknell, P. A., Leach, M. C., and Rowe, C. (2018). Handling method alters the hedonic value of reward in laboratory mice. Sci. Rep. 8:2448. doi: 10.1038/s41598-018-20716-3

Cohen, J. D., and Castro-Alamancos, M. A. (2010). Neural correlates of active avoidance behavior in superior colliculus. J. Neurosci. 30, 8502-8511. doi: 10.1523/JNEUROSCI.1497-10.2010

De Franceschi, G., Vivattanasarn, T., Saleem, A. B., and Solomon, S. G. (2016). Vision guides selection of freeze or flight defense strategies in mice. Curr. Biol. 26, 2150-2154. doi: 10.1016/j.cub.2016.06.006

Deacon, R. M. J. (2006). Housing, husbandry and handling of rodents for behavioral experiments. Nat. Protoc. 1, 936-946. doi: 10.1038/nprot.2006.120

Ebisch, S. J. H., Salone, A., Martinotti, G., Carlucci, L., Mantini, D., Perrucci, M. G., et al. (2016). Integrative processing of touch and affect in social perception: an fMRI study. Front. Hum. Neurosci. 10:209. doi: 10.3389/fnhum.2016.00209

Ellingsen, D. M., Wessberg, J., Chelnokova, O., Olausson, H., Laeng, B., and Leknes, S. (2014). In touch with your emotions: oxytocin and touch change social impressions while others' facial expressions can alter touch. Psychoneuroendocrinology 39, 11-20. doi: 10.1016/j.psyneuen.2013.09.017

Evans, D. A., Stempel, A. V., Vale, R., Ruehle, S., Lefler, Y., and Branco, T. A. (2018). synaptic threshold mechanism for computing escape decisions. Nature 558, 590-594. doi: 10.1038/s41586-018-0244-6

Fenoglio, K. A. (2006). Neuroplasticity of the hypothalamic-pituitary-adrenal axis early in life requires recurrent recruitment of stress-regulating brain regions. J. Neurosci. 26, 2434-2442. doi: 10.1523/JNEUROSCI.4080-05.2006

Field, T., Hernandez-Reif, M., Diego, M., Schanberg, S., and Kuhn, C. (2005). Cortisol decreases and serotonin and dopamine increase following massage therapy. Int. J. Neurosci. 115, 1397-1413. doi: 10.1080/00207450590956459

Gdalyahu, A., Tring, E., Polack, P. O., Gruver, R., Golshani, P., Fanselow, M. S., et al. (2012). Associative fear learning enhances sparse network coding in primary sensory cortex. Neuron 75, 121-132. doi: 10.1016/j.neuron.2012.04.035
91632303, and 31600860), the International Partnership Program of Chinese Academy of Sciences (Grant No. 172644KYS820170004), the External Cooperation Program of the Chinese Academy of Sciences (Grant No. GJHZ1508), the Guangdong Provincial Key Laboratory of Brain Connectome and Behavior (Grant No. 2017B030301017), the Shenzhen Municipal People's Government (Grant Nos. JCYJ20150529143500959 and JCYJ20160429190927063), the Shenzhen Discipline Construction Project for Neurobiology DRCSM (Grant No. [2016]1379), Ten Thousand Talent Program, and the Helmholtz-CAS Joint Research Grant, GJHZ1508, and also supported by Guangdong Special Support Program.

Ghitani, N., Bayguinov, P. O., Vokoun, C. R., McMahon, S., and Jackson, M. B. (2014). Excitatory synaptic feedback from the motor layer to the sensory layers of the superior colliculus. J. Neurosci. 34, 6822-6833. doi: 10.1523/JNEUROSCI. 3137-13.2014

Grandin, T. (1997). Assessment of stress during handling and transport. J. Anim. Sci. 75, 249-257. doi: 10.2527/1997.751249x

Harlow, H., and Suomi, S. (1970). The nature of love. Am. Psychol. 25, 161-168. doi: $10.1037 / \mathrm{h} 0029383$

Huang, L., Yuan, T., Tan, M., Xi, Y., Hu, Y., Tao, Q., et al. (2017). A retinoraphe projection regulates serotonergic activity and looming-evoked defensive behaviour. Nat. Commun. 8:14908. doi: 10.1038/ncomms14908

Hurst, J. L., and West, R. S. (2010). Taming anxiety in laboratory mice. Nat. Methods 7, 825-826. doi: 10.1038/nmeth.1500

Janak, P. H., and Tye, K. M. (2015). From circuits to behaviour in the amygdala. Nature 517, 284-292. doi: 10.1038/nature14188

Koutsikou, S., Watson, T. C., Crook, J. J., Leith, J. L., Lawrenson, C. L., Apps, R., et al. (2015). The periaqueductal gray orchestrates sensory and motor circuits at multiple levels of the neuraxis. J. Neurosci. 35, 14132-14147. doi: 10.1523/ JNEUROSCI.0261-15.2015

LeDoux, J. (2012). Rethinking the emotional brain. Neuron 73, 653-676. doi: 10.1016/j.neuron.2012.02.004

Li, L., Feng, X. L., Zhou, Z., Zhang, H. Q., Shi, Q. Q., Lei, Z. G., et al. (2018). Stress accelerates defensive responses to looming in mice and involves a locus coeruleus-superior colliculus projection. Curr. Biol. 28, 859.e5-871.e5. doi: 10. 1016/j.cub.2018.02.005

Lumpkin, E. A., Marshall, K. L., and Nelson, A. M. (2010). The cell biology of touch. J. Cell Biol. 191, 237-248. doi: 10.1083/jcb.201006074

Macaluso, E., Frith, C. D., and Driver, J. (2000). Modulation of human visual cortex by crossmodal spatial attention. Science 289, 1206-1208. doi: 10.1126/science. 289.5482.1206

Macaluso, E., Frith, C. D., and Driver, J. (2002). Crossmodal spatial influences of touch on extrastriate visual areas take current gaze direction into account. Neuron 34, 647-658. doi: 10.1016/S0896-6273(02)00678-5

Madruga, C., Xavier, L. L., Achaval, M., Sanvitto, G. L., and Lucion, A. B. (2006) Early handling, but not maternal separation, decreases emotional responses in two paradigms of fear without changes in mesolimbic dopamine. Behav. Brain Res. 166, 241-246. doi: 10.1016/j.bbr.2005.08.005

Mooney, R. D., Huang, X., and Rhoades, R. W. (1992). Functional influence of interlaminar connections in the hamster's superior colliculus. J. Neurosci. 12, 2417-2432. doi: 10.1523/JNEUROSCI.12-06-02417.1992

Morgan, C. L. (1896). Habit and Instinct. London: Edward Arnold Publishers. doi: 10.1037/12922-000

Muijres, F. T., Elzinga, M. J., Melis, J. M., and Dickinson, M. H. (2014). Flies evade looming targets by executing rapid visually directed banked turns. Science 344, 172-177. doi: 10.1126/science. 1248955

Newman, M. E. J. (2006). Modularity and community structure in networks. Proc. Natl. Acad. Sci. U.S.A. 103, 8577-8582. doi: 10.1073/pnas.0601602103

Peled-Avron, L., Perry, A., and Shamay-Tsoory, S. G. (2016). The effect of oxytocin on the anthropomorphism of touch. Psychoneuroendocrinology 66, 159-165. doi: 10.1016/j.psyneuen.2016.01.015 
Penzo, M. A., Robert, M., Tucciarone, J., Bundel, D. D., Wang, M. H., Aelst, L. V., et al. (2015). The paraventricular thalamus controls a central amygdala fear circuit. Nature 519, 455-459. doi: 10.1038/nature 13978

Pernon, E., Pry, R., and Baghdadli, A. (2007). Autism: tactile perception and emotion. J. Intellect. Disabil. Res. 51, 580-587. doi: 10.1111/j.1365-2788.2006. 00931.x

Poole, T. (1997). Happy animals make good science. Lab. Anim. 31, 116-124. doi: $10.1258 / 002367797780600198$

Power, J. D., Schlaggar, B. L., Lessov-Schlaggar, C. N., and Petersen, S. E. (2013). Evidence for hubs in human functional brain networks. Neuron 79, 798-813. doi: 10.1016/j.neuron.2013.07.035

Ratcliffe, M. (2012). What is Touch?? Australas. J. Philos. 90, 413-432. doi: 10.1080/ 00048402.2011 .598173

Robertson, C. E., and Baron-Cohen, S. (2017). Sensory perception in autism. Nat. Rev. Neurosci. 18, 671-684. doi: 10.1038/nrn.2017.112

Rogers-Carter, M. M., Varela, J. A., Gribbons, K. B., Pierce, A. F., McGoey, M. T., Ritchey, M., et al. (2018). Insular cortex mediates approach and avoidance responses to social affective stimuli. Nat. Neurosci. 21, 404-414. doi: 10.1038/ s41593-018-0071-y

Rubinov, M., and Sporns, O. (2010). Complex network measures of brain connectivity: uses and interpretations. NeuroImage 52, 1059-1069. doi: 10. 1016/j.neuroimage.2009.10.003

Sahibzada, N., Dean, P., and Redgrave, P. (1986). Movements resembling orientiation or avoidance elicited by electrical stimulation of the superior colliculus in rats. J. Neurosci. 6, 723-733. doi: 10.1523/JNEUROSCI.06-0300723.1986

Saito, Y., and Isa, T. (2005). Organization of interlaminar interactions in the rat superior colliculus. J. Neurophysiol. 93, 2898-2907. doi: 10.1152/jn.01051.2004

Salay, L. D., Ishiko, N., and Huberman, A. D. (2018). A midline thalamic circuit determines reactions to visual threat. Nature 557, 183-189. doi: 10.1038/ s41586-018-0078-2

Saper, C. B. (2002). The central autonomic nervous system: conscious visceral perception and autonomic pattern generation. Annu. Rev. Neurosci. 25, 433469. doi: 10.1146/annurev.neuro.25.032502.111311

Scheele, D., Kendrick, K. M., Khouri, C., Kretzer, E., Schläpfer, T. E., Stoffel-Wagner, B., et al. (2014). An oxytocin-induced facilitation of neural and emotional responses to social touch correlates inversely with autism traits. Neuropsychopharmacology 39, 2078-2085. doi: 10.1038/npp. 2014.78

Shang, C. P., Chen, Z. J., Liu, A. X., Li, Y., Zhang, J. J., Qu, B. L., et al. (2018). Divergent midbrain circuits orchestrate escape and freezing responses to looming stimuli in mice. Nat. Commun. 9, 1-17. doi: 10.1038/s41467-01803580-7
Shang, C. P., Liu, Z. Y., Chen, Z. J., Shi, Y. C., Wang, Q., Liu, S., et al. (2015). A parvalbumin-positive excitatory visual pathway to trigger fear responses in mice. Science 348, 1472-1477. doi: 10.1126/science.aaa8694

Soares, M. C., Oliveira, R. F., Ros, A. F., Grutter, A. S., and Bshary, R. (2011). Tactile stimulation lowers stress in fish. Nat. Commun. 2:534. doi: 10.1038/ ncomms 1547

Stein, B. E. (1984). Development of the superior colliculus. Annu. Rev. Neurosci. 7, 95-125. doi: 10.1146/annurev.ne.07.030184.000523

Temizer, I., Donovan, J. C., Baier, H., and Semmelhack, J. L. (2015). A visual pathway for looming-evoked escape in larval zebrafish. Curr. Biol. 25, 18231834. doi: 10.1016/j.cub.2015.06.002

Tovote, P., Fadok, J. P., and Lüthi, A. (2015). Neuronal circuits for fear and anxiety. Nat. Rev. Neurosci. 16, 317-331. doi: 10.1038/nrn3945

Vale, R., Evans, D. A., and Branco, T. (2017). Rapid spatial learning controls instinctive defensive behavior in mice. Curr. Biol. 27, 1342-1349. doi: 10.1016/ j.cub.2017.03.031

Vetere, G., Kenney, J. W., Tran, L. M., Xia, F., Steadman, P. E., Parkinson, J., et al. (2017). Chemogenetic interrogation of a brain-wide fear memory network in mice. Neuron 94, 363.e4-374.e4. doi: 10.1016/j.neuron.2017.03.037

Wei, P., Liu, N., Zhang, Z. J., Liu, X. M., Tang, Y. Q., He, X. B., et al. (2015). Processing of visually evoked innate fear by a non-canonical thalamic pathway. Nat. Commun. 6:6756. doi: 10.1038/ncomms7756

Wurtz, R. H., and Goldberg, M. E. (1971). Superior colliculus cell responses related to eye movements in awake monkeys. Science 171, 82-84. doi: 10.1126/science. 171.3966 .82

Yilmaz, M., and Meister, M. (2013). Rapid innate defensive responses of mice to looming visual stimuli. Curr. Biol. 23, 2011-2015. doi: 10.1016/j.cub.2013.08.015

Zhao, X. Y., Liu, M. N., and Cang, J. H. (2014). Visual cortex modulates the magnitude but not the selectivity of looming-evked responses in the superior colliculus of awake mice. Neuron 81, 202-213. doi: 10.1016/j.neuron.2014.08.037

Conflict of Interest Statement: The authors declare that the research was conducted in the absence of any commercial or financial relationships that could be construed as a potential conflict of interest.

Copyright (c) 2018 Liu, Chen, Liu, Wang, Huang, Wang and Wang. This is an open-access article distributed under the terms of the Creative Commons Attribution License (CC BY). The use, distribution or reproduction in other forums is permitted, provided the original author(s) and the copyright owner(s) are credited and that the original publication in this journal is cited, in accordance with accepted academic practice. No use, distribution or reproduction is permitted which does not comply with these terms. 